pediatric autoimmune neuropsychiatric disorders associated with streptococcal infection (PANDAS) and their limitations (Neurology June 1998;50:1530-1534). Criteria for diagnosis of TS-PANDAS include childhood onset of tics, and sudden onset or exacerbations related to streptococcal infection.

\title{
TOURETTE SYNDROME PREVALENCE
}

The prevalence of Tourette syndrome in a mainstream school population (ages 13 to 14 years) in West Essex, England has been determined by parent, teacher, and pupil questionnaires, class observations to identify tics, and subject and parent face-to-face interviews, and reported from the Section of Epidemiology and General Practice, Institute of Psychiatry, Denmark Hill, London, UK. From data available on 166 pupils in one school year, 5 children had TS, based on DSM-III-R criteria. Hyperactivity was an associated disorder in 4 and ADHD was present in 1. The prevalence estimate for TS in this age group was 299 per 10,000 pupils, or $3 \%$. In comparison, the clinical records of the West Essex child and adolescent psychiatry service revealed 18 referred cases of TS out of a population of 37,500 children, ages 4 to 16 years, or a prevalence rate of 4.8 per 10,000 $(0.05 \%)$. (Mason A, Banerjee S, Eapen V, Zeitlin H, Robertson MM. The prevalence of Tourette syndrome in a mainstream school population. Dev Med Child Neurol June 1998;40:292-296). (Respond: Dr Sube Banerjee, Section of Epidemiology and General Practice, The Institute of Psychiatry, De Crespigny Park, Denmark Hill, London SE5 8AF, UK).

COMMENT. TS among children in a community as a whole is more common and milder than that diagnosed in a health-care psychiatry service. The relative prevalence rates are $3 \%$ in a school population compared to $0.05 \%$ in a child psychiatry service in the UK. Prior studies involving school children from Monroe County, NY and a California school district found estimated TS prevalence rates of 3 per $10,000(0.03 \%)$ and 76 per $10,000(0.8 \%)$, respectively. (see Progress in Pediatric Neurology I, PNB Publ, 1991;pp228-9). The prevalence of TS in the UK study is four times greater than the highest US estimate in similar populations. The authors attribute the higher TS prevalence to be related to more thorough case ascertainment methods.

\section{TOURETTE SYNDROME MATURATIONAL CHANGES}

Tourette syndrome is considered a model neuropsychiatric disorder of childhood, reflecting an interaction between genetic and environmental factors, in a review of clinical characteristics, heredity and vulnerability, and neuroanatomy and neurochemistry, from the Child Study Center, Yale University, New Haven, CT. Referring to Israeli studies, the prevalence of TS is cited at $0.1 \%$ in boys and $0.01 \%$ in girls, or 1 in 1500 children; milder forms or chronic tic disorders have much higher prevalence rates of 4-6\%. Most children developing tics have a history of prior hyperactivity or ADHD, and $30-60 \%$ develop OCD in preadolescence. Improvement during adulthood can be expected. TS is often familial, about $8 \%$ of relatives having TS and $17 \%$ exhibiting chronic tic disorders. Degree of severity is broad, mostly mild and only a minority showing serious functional impairment. An autosomal dominant transmission of inheritance is suggested by studies of large, multigenerational pedigrees, with higher penetrance for males than females. The search for the gene locus has been elusive, impeding research in the relative importance of genetic and environmental factors. Anatomically, symptoms of TS are related to abnormalities in cortico-striatal-thalamic-cortico pathways. Chemically, dopaminergic and serotonergic systems may be involved. (Cohen DJ, Leckman JF, Pauls D. Neuropsychiatric disorders of childhood: Tourette's syndrome as a model. Acta 